\title{
DIABETES \\ DNA METHYLATION AFFECTS T2DM RISK
}

DNA methylation patterns might explain the increased risk of developing type 2 diabetes mellitus (T2DM) in people of Indian Asian descent compared with those of European descent, according to new findings.

Differences in the principle risk factors for T2DM (diet, obesity and reduced physical activity) do not explain the increased incidence of T2DM in people of Indian Asian descent. Previous work has shown that methylation of DNA, a key mechanism in regulation of gene expression, is altered in patients with obesity and insulin resistance. This finding led a group of researchers to investigate whether differences in DNA methylation could predict the incidence of T2DM and explain the different risk profiles of people of Indian Asian or European descent.

John Chambers, Jaspal Kooner and colleagues carried out a case-control study including 25,372 participants in the London Life Sciences Prospective Population study. Over a follow-up period of 8 years, $11.9 \%$ of the Indian Asian individuals and $4.3 \%$ of the European participants developed T2DM. The investigators conducted epigenomewide association analyses of blood samples from Indian Asian participants with incident T2DM and Indian Asian control individuals who were matched for age and sex. Replication of the top-ranking variants was then carried out with samples from the European participants.

Methylation at five loci (ABCG1, PHOSPHO1, SOCS3, SREBF1 and TXNIP) was associated with an increased risk of developing T2DM, independently of established risk factors. Furthermore, the DNA methylation patterns differed between participants of Indian Asian descent and those of European descent. Overall, participants of Indian Asian descent had higher methylation scores than European participants. The authors of the paper suggest that measuring DNA methylation might help to explain the increased risk of T2DM in people of Indian Asian descent.

Why an association exists between methylation at these loci and an increased risk of T2DM is unclear. However, "DNA methylation might be a biomarker of unfavourable patterns of adiposity and insulin resistance," write the authors.

Claire Greenhill

Original article Chambers, J. C. et al. Epigenome-wide association of DNA methylation markers in peripheral blood from Indian Asians and Europeans with incident type 2 diabetes: a nested case-control study. Lancet Diabetes Endocrinol. doi:10.1016/S2213-8587(15)00127-8 